\title{
FRATs: Searching for Fast Radio Transients in Real Time with LOFAR
}

\section{S. ter Veen, P. Schellart, and H. Falcke for the LOFAR Transients and Cosmic Ray Key Science Projects}

Department of Astrophysics, IMAPP, Radboud University Nijmegen, 6500 GL Nijmegen, NL

email: s.terveen@astro.ru.nl

\begin{abstract}
The aim of the FRATs project is to detect single dispersed pulses from Fast Radio Transients with LOFAR in real time. The pulses can originate from pulsars, RRATS and other classes of known or unknown objects. To detect the pulses a trigger algorithm is run on an incoherent beam from the different LOFAR stations. The beam has a wide field of view and can be formed parallel to other observations. A precise localisation is achieved by storing and processing off-line the data from each dipole, giving all-sky coverage with a spatial resolution of the order of arc-seconds. The source is identified by making high-time-resolution images. The method has been tested by detecting and identifying a giant pulse from the Crab pulsar.
\end{abstract}

Keywords. techniques: interferometric, (stars:) pulsars: general

\section{Introduction}

The most well-known radio transients are pulsars. Detecting more pulsars will assist in studies of the neutron-star population in the Galaxy and in globular clusters, and in understanding the emission mechanism of these extreme objects. In the pioneering years pulsars were found by looking for single pulses, but later on periodicity searches were usually used because they offered higher sensitivity. Single-pulse searches have once again become popular, and have led to the discovery of Rotating Radio Transients (RRATS) (McLaughlin et al. 2006) - pulses that repeat only occasionally. What objects give those sporadic pulses, and how many more are there? If the pulses originate from pulsars they can be giant pulses from distant normal pulsars, or can originate in almost dead, nearby pulsars that only emit rarely. Other suggestions regarding the origin of sporadic pulses include exoplanets with an emission mechanism similar to the Jupiter-IO system. To hunt for those (and for any other kind of pulses) we developed a real-time detection mode for LOFAR (the LOw Frequency Array; see Stappers et al. 2011).

\section{Method}

The difficulty of detecting strong single pulses is that they occur only occasionally, so a large instantaneous sky coverage and observing time are therefore required. Furthermore, when only a single pulse is found, one wants to be absolutely sure that it is from an astrophysical source and not a terrestrial one. For example, it is still undecided what was the origin of the Lorimer burst (Lorimer et al. 2007). A precise position and correction for dispersion are also required. We explain how LOFAR meets those requirements within the Fast Radio Transients (FRATs) project. 


\subsection{LOFAR}

LOFAR is a new-generation distributed digital radio telescope. Instead of using large dishes, it uses stations with fields of small dual polarization antennæ. Most of those stations are located in the Netherlands, but there are also ones in Germany, France, the UK and Sweden. There are two types of antennæ: Low Band Antennæ (10-90 MHz) and High Band Antennæ (110-240 MHz). The stations are placed on base-lines from $100 \mathrm{~m}$ to $1000 \mathrm{~km}$. There are three observating modes: imaging, beam-forming, and storing the data per dipole. Data are stored in the memory ring-buffers of the Transient Buffer Boards (TBBs) at a sample time of $5 \mathrm{~ns}$. Currently 1.3 seconds of raw data can be stored, but an increase to 5.2 seconds is planned. Buffer-time can be traded for bandwidth or, by storing fewer dipoles, for sensitivity. If an interesting event is found, the buffers can be stopped and read out, partially or entirely.

The beam-forming mode corrects for the delays between the different receiving elements, in the first stage in each station and in the second stage between the stations. The latter can be done either coherently (added in phase, small field of view (FoV), high sensitivity) or incoherently (added in power, large FoV (10-18 deg. ${ }^{2} @ 150 \mathrm{MHz}$ ), lower sensitivity). The total bandwidth in this mode is $48 \mathrm{MHz}$, but it can be split for observing in many different directions simulteneously. The beam-formed data consist of many channels with a high frequency- and time-resolution $(700 \mathrm{~Hz}-12 \mathrm{kHz} ; 0.08-1.3 \mathrm{~ms})$.

\subsection{Real-time detection}

To detect pulses we use the incoherently summed beam-formed data from all active stations, taken in parallel to an imaging observation. That meets the requirements of a large FoV and a long observation time. The trigger algorithm that processes the data first has to correct for dispersion, i.e. the delay caused by the interstellar medium and making signals of a lower frequency arrive later, according to the relation $\Delta t_{D M}=$ $4.15 \mathrm{~ms} \mathrm{DM}\left(\nu_{1, \mathrm{GHz}}^{-2}-\nu_{2, \mathrm{GHz}}^{-2}\right)$; that is done in several frequency bands ( $\sim \mathrm{MHz}$ bandwidth). Narrow-band radio-frequency interference (RFI) may trigger only one band but a real signal will trigger multiple bands. It corrects for the time delay between the $\sim \mathrm{kHz}$ channels in each band for several dispersion measure trials (DM), and checks for peaks using a simple $P>S_{\text {sys }}+N * \Delta S$ algorithm, where $P$ is the power of the signal, $S_{\text {sys }}$ is calculated by taking the mean, and $\Delta S$ is the standard deviation of the de-dispersed data. The algorithm then checks for a coincidence between peaks found in the different bands corresponding to the expected delay for the given DM (Fig. 1). If such an event is found, a trigger message is sent to obtain the TBB data.

\subsection{Offline localisation}

The TBB data are used to find the direction of the pulse; they contain the raw data from each available dipole, and beams can be formed from them in any direction at the full resolution capabilities of LOFAR. They can, for instance, be used to image the whole sky (LBA), or $2000 \operatorname{deg}^{2}$ (HBA). Near-field beam-forming is used to discriminate against terrestrial signals (and also to study cosmic-ray air showers and lightning).

To investigate where the pulse came from, incoherent beams are formed in all directions to check whether it originated in the pointing direction or in a sidelobe. Coherent beams are formed in the actual direction of the pulse to find its precise location; a precision of $<1$ arcsecond resolution is expected. Fig. 1 shows the de-dispersed data in four frequency bands of a giant pulse for which a trigger was issued and TBB data were obtained. Fig. 2 shows the de-dispersed data of a pulse imaged from those TBB data. 
A pulse from the Crab pulsar in 4 frequency bands

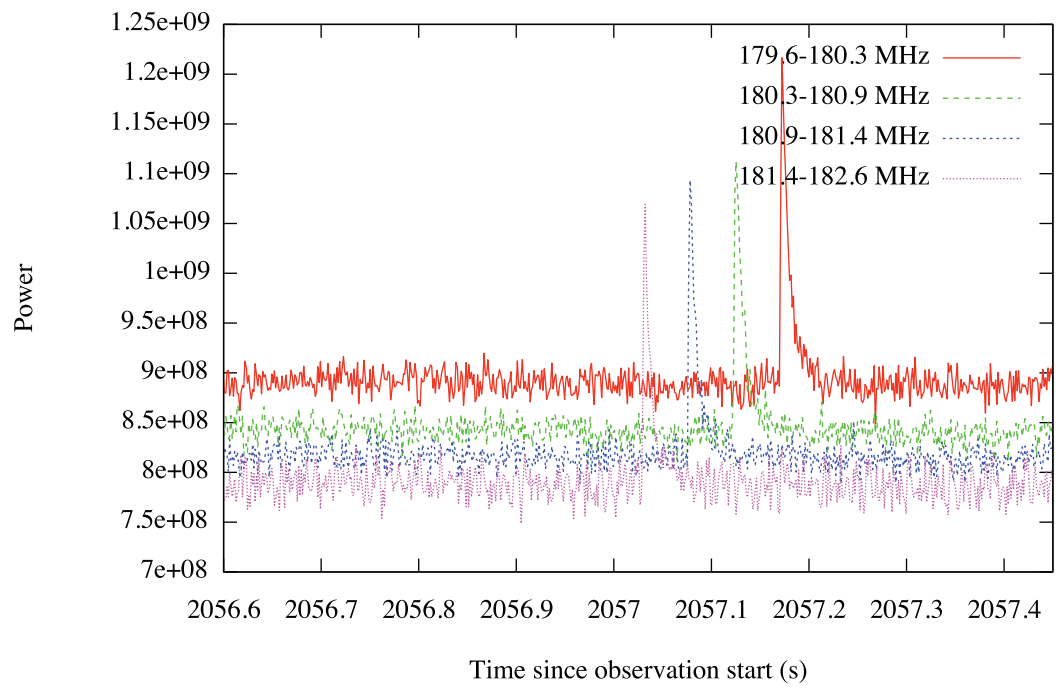

Figure 1. A pulse from the Crab pulsar, de-dispersed in each of 4 frequency bands. The time delay between the different pulses is caused by dispersion.

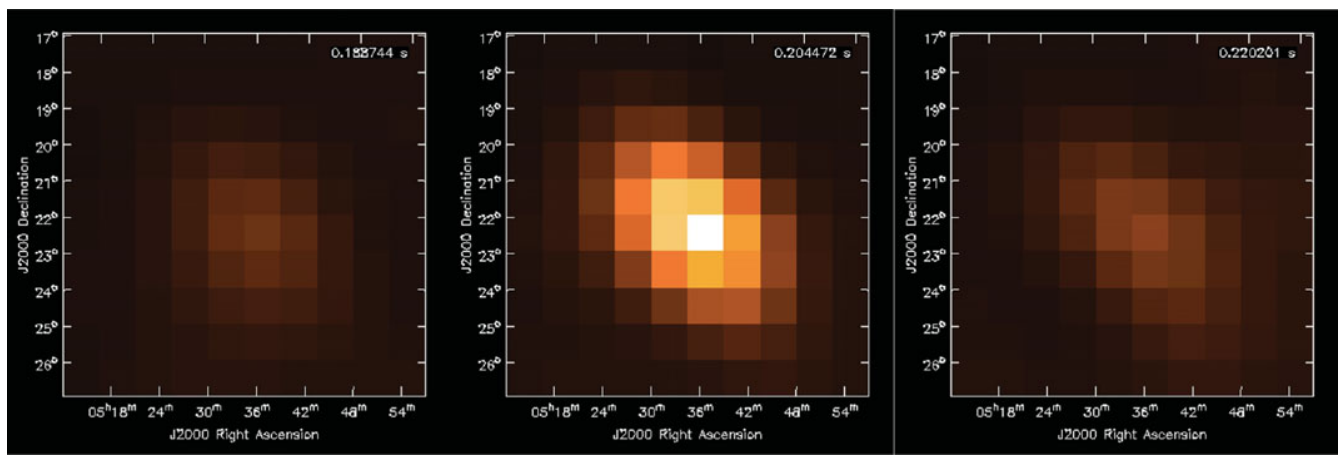

Figure 2. A de-dispersed image in the direction of the Crab pulsar before, during and after the pulse. (Made from the TBB data from only one LOFAR station, hence the inferior resolution).

\section{Conclusion}

A real-time search for Fast Radio Transients is feasible with LOFAR. By using an incoherent beam, parallel to other observations, large sky and time coverage are obtained. To refine the direction and discriminate against RFI the raw data from each dipole are obtained and analyzed. A prototype of this pipeline has run succesfully, proving the concept and pointing the way to a new horizon in time-domain radio astronomy.

\section{References}

McLaughlin, M. A., et al. 2006, Nature, 439, 817

Stappers, B., et al. 2011, A\&A, 530, A80

Lorimer, D. R., Bailes, M., McLaughlin, M. A., Narkevic, D. J., \& Crawford, F. 2007, Science, 318,777 